\title{
Painful recall in elective electrical cardioversion with propofol and the need for additional analgesia
}

\author{
Van Winden DFM ${ }^{*}$, Westra $\mathrm{A}^{1 *}$, Dennessen $\mathrm{PJW}^{2}$, Monnink SHJ ${ }^{3}$, Verdouw $\mathrm{BC}^{4}$ and R le Kluse ${ }^{3}$ \\ ${ }^{1}$ Department of Emergency Medicine, Reinier de Graaf hospital, The Netherlands \\ ${ }^{2}$ Department of Intensive Care, Haaglanden Medical Center, The Netherlands \\ ${ }^{3}$ Department of Cardiology, Reinier de Graaf hospital, The Netherlands \\ ${ }^{4}$ Department of Anaesthesiology, Reinier de Graaf hospital, The Netherlands
}

\begin{abstract}
Introduction: Electrical cardioversion $(\mathrm{ECV})$ is a short but painful procedure for treating cardiac dysrhythmias. There is a wide variation regarding the medication strategy to facilitate this procedure. Many different sedative techniques for ECV are described. Currently, the optimal medication strategy to prevent pain in ECV has yet to be established. The role for additional analgesic agents to prevent pain during the procedure remains controversial and evidence is limited.

Methods: We conducted a prospective multicenter study to determine the incidence of painful recall in ECV with propofol as a sole agent for sedation, in order to assess the indication for additional opioids. In all patients sedation was induced with propofol titrated till loss of eyelash reflex and non-responsiveness to stimuli, corresponding to Ramsay Sedation Score level 5-6. ECV was performed with extracardiac biphasic electrical shocks. The primary outcome was painful recall of the procedure, defined as numeric pain rating scale (NRS) $\geq 4$. Secondary outcome parameters were pain at the side of the defipads and muscle pain after ECV.

Results: A total of 226 patients were enrolled in this study. Six patients were excluded due to missing date or violation of study protocol. One patient (0.4\%) reported recall of the procedure and NRS 7 and two patients $(0.9 \%)$ NRS $1-3$ without recall of the procedure. Complete amnesia was observed in 223 patients, with NRS 0 . The mean of the total dose of propofol was $1.1 \mathrm{mg} / \mathrm{kg}$. Fifteen patients $(6.4 \%)$ experienced pain at the side of the defipads and six patients (0.9\%) complained of muscle pain after the procedure.

Conclusions: In this prospective multicenter study, painful recall (NRS $\geq 4$ ) in ECV was found in $0.4 \%$ of the patients. Propofol as a sole agent provided effective sedation and retrograde amnesia in $99.6 \%$ of the patients. Our findings support that additional opioids to propofol sedation in ECV are not indicated to prevent painful recall.
\end{abstract}

\section{Background}

External electrical cardioversion (ECV) is a short and painful procedure for treating cardiac dysrhythmias. There is a wide variation regarding the medication strategy to facilitate ECV. The practice of cardioversion varies between clinicians and countries and involves use of a sedative agent (such as propofol, etomidate, thiopentone or methohexital) or a sedative agent (such as midazolam or diazepam) with or without additional analgesia [1]. Factors that influence the choice of drug include speed of action, recovery time, adverse effects caused by the drug and patient recall or awareness of pain.

Propofol is a sedative with amnestic properties [1-3]. Limited previous studies have addressed awareness or recall in ECV with propofol. Incidences varied from 0-23\% [3-15]. These studies had severe limitations; recall was not the primary outcome measure, findings were based on small patient groups, results were not differentiated for the sedatives used, supplemental opioids were given, the studies described a variety of short painful procedures or did not reflect clinical practice.

Currently, the optimal medication strategy to prevent pain in ECV has yet to be established. The evidence for the use of analgesic agents such as opioids in conjunction with sedatives to prevent pain is limited and controversial. The aim of this study was to determine the incidence of painful recall in ECV using propofol as the sole agent for sedation and to assess the need for additional analgesia.

\section{Methods}

\section{Study design}

This was a prospective multicenter study that took place from December 2014 to November 2016 at the Intensive Care Unit (ICU) of Haaglanden Medical Centre and the Cardiac Care Unit (CCU) of Reinier de Graaf Hospital, the Netherlands. In these departments, sedation is performed at the discretion of the attending Intensive Care specialist or anesthesiologists. Propofol is the sedating agent of choice in ECV. Additional opioids are usually not given.

The primary outcome was the incidence of painful recall of the ECV after sedation with propofol. Secondary outcomes were pain at the side of the defipads and muscle pain after the procedure.

*Correspondence to: AAnne-Maayke Westra, Department of Emergency Medicine, Reinier de Graaf hospital 2625 AD Delft, The Netherlands, Tel: 0031 15260 3385, Fax: 003115260 3806, E-mail: a.westra@rdgg.nl

D.F.M. van Winden, Department of Emergency Medicine, Reinier de Graaf hospital 2625 AD Delft, The Netherlands, E-mail: dfm@vanwinden.net

Key words: electrical cardioversion, pain, recall, anesthesia, propofol

Received: March 09, 2018; Accepted: March 21, 2018; Published: March 24, 2018 


\section{Patients}

All patients (aged 18 years or older) scheduled for cardioversion for arrhythmias such as atrial fibrillation, atrial flutter or supraventricular tachycardia with an indication for ECV were eligible for study participation. Patients were excluded in case of hypersensitivity to propofol. The regional Medical Ethics Committee Zuidwest Holland, in the Netherlands exempted this study for formal ethical approval and patient consent. However, verbal consent was asked to all potential study participants before participation in the study. The trial is registered at www.trialregister.nl

\section{Procedure}

Depending on the clinical practice of the treating specialist, some patients received a small dose of lidocaine to prevent pain due to injection of propofol. No additional analgesics were given. Anesthesia for ECV was induced with propofol. Propofol was titrated with physician's choice of dose till adequate sedation was achieved. Adequate sedation was defined as a state of unconsciousness with non-responsiveness to stimuli and loss of eyelash reflex, corresponding with Ramsay Sedation Scale level 5 or 6 . The level of sedation was measured by the attending ICU specialist or anesthesiologists. In case of inadequate sedation, additional boluses of propofol were given as often as necessary.

ECV was performed by the cardiologist with extracardiac biphasic electrical shocks ranging from 50 to 200 Joules. If sinus rhythm was not restored, more shocks were delivered.

After the patients had returned to their baseline mental status, they were asked to report any pain or recall associated with the procedure using the Numeric Rating scale (NRS). The NRS score is a validated score to express the intensity of pain by choosing a number, from 0 (no pain) to 10 (worst pain imaginable). NRS $\geq 4$ is used to refer to inadequately treated pain [16]. In this study we also used NRS $\geq 4$ to define the presence of painful recall. Besides painful recall, patients were also asked about local pain during injection of propofol, pain at the side of the defipads and muscle pain after cardioversion.

Records were kept of the pain scores, age, sex, weight, propofol doses, the use of other analgesics, the number of shocks, total amount of energy used. Data were collected by the ICU specialist, the ICU resident or the CCU nurse on a pre-formatted data sheet during the procedure.

\section{Safety}

All patients were preoxygenated with $100 \%$ oxygen via a nonrebreathing facemask while breathing spontaneously before induction of anesthesia. Cardiorespiratory parameters were monitored continuously during and after the procedure until patients had returned to their baseline mental status. In case of upper airway obstruction, a chin lift and jaw thrust manoeuvre was applied. If apnea occurred during the procedure, patients were manually ventilated with $100 \%$ oxygen. Adverse events and complications were recorded in the patient files but are not described in this study as they fall beyond the scope of this present study.

\section{Statistical analysis}

As this was a descriptive study, no power calculation was undertaken. Statistical analysis was performed using SPSS Statistics v. 17.0. Standard descriptives were obtained. Outcomes in this study included dichotomous outcomes (recall, pain at the side of defipads, muscle pain) and continuous data (NRS, number of shocks). Values were expressed as mean \pm standard deviation or percentages of patients, as appropriate.

\section{Results}

\section{Patients}

A total of 226 patients who underwent ECV facilitated with propofol as the sedating agent were enrolled in the study. Six patients were excluded because of missing data. The mean age was $68.7 \pm 10.5$ years, range 30 to 93 years and 148 $(65,5 \%)$ of the patients were male. The baseline characteristics of the patients are shown in table 1 .

Sixty-two (27.4\%) patients received a single dose of lidocaine to numb the blood vessel before administration of propofol. The median propofol dose required was $1.1 \mathrm{mg} / \mathrm{kg}$. The dose varied with a inter quartile range $0.37 \mathrm{mg} /$ $\mathrm{kg}$ (Table 1).

Complete amnesia was observed in 223 patients (98.7\%) with NRS 0. One patient $(0.4 \%)$ had reported painful recall of the procedure with NRS 7 and recollection of the two shocks. This patient received $10 \mathrm{mg}$ lidocaine to numb the bloodvessel and $90 \mathrm{mg}$ propofol $(0.83 \mathrm{mg} / \mathrm{kg})$. The patient was adequately sedated with loss of eyelash reflex and non-responsiveness to auditory stimulus.

We also studied the need for simple analgesics after ECV. Fifteen patients experienced a burning sensation or pain at the side of the defipads and six patients complained from muscle pain after the ECV. One, two or three shocks were needed in respectively 178,34 and 14 patients. The patients that received 3 shocks reported no pain with NRS 0 after the procedure. Findings of the procedure are presented in Table 2.

\section{Discussion}

\section{Studies addressing recall}

The aim of this study was to determine the incidence of painful recall in ECV using propofol as the sole agent for sedation. In this multicenter study painful recall of the ECV was found in $0.4 \%$ of the patients.

As far as we know this is the first study that evaluates the amnesic properties of propofol in ECV as a primary outcome measure in a large consecutive patient group. The limited previous studies that adressed painful recall after sedation with propofol described incidences of recall varying from $0-23 \%$ in a variety of short painful procedures

Table 1. Patient Characteristics of the study subjects and doses of propofol and analgesic agents

\begin{tabular}{|l|l|}
\hline Age $($ years $)$, mean $($ range $)$ & $68.7(30-93)$ \\
\hline Weight $(\mathrm{kg})$, mean $( \pm \mathrm{SD})$ & $86.5 \pm 17.7$ \\
\hline Length $(\mathrm{cm})$, mean $( \pm \mathrm{SD})$ & $177.9 \pm 9.9$ \\
\hline Lean body mass $(\mathrm{kg})$, mean $( \pm \mathrm{SD})$ & $61.6 \pm 12.4$ \\
\hline Gender $($ male/female) \% & $148 / 78(65.5 / 34.5)$ \\
\hline Total dose of propofol $(\mathrm{mg})$, mean & $90.8 \pm 37.6$ \\
\hline Dose of propofol/weight $(\mathrm{mg} / \mathrm{kg})$, median $( \pm \mathrm{SD})$ & $1.1 \pm 0.3$ \\
\hline Dose of propofol/lean body weight $(\mathrm{mg} / \mathrm{kg})$, mean $( \pm \mathrm{SD})$ & $1.5 \pm 0.7$ \\
\hline Number of patients given lidocaine $(\mathrm{n}, \%)$ & $62(27.4)$ \\
\hline
\end{tabular}

Table 2. Details of findings of the ECV

\begin{tabular}{|l|l|}
\hline $\begin{array}{l}\text { Number of shocks (n/N) (\%) } \\
1\end{array}$ & $178(76.7)$ \\
2 & $34(15.0)$ \\
3 & $14(6.2)$ \\
\hline Reported amnesia NRS 0 & $223(98.7)$ \\
\hline Reported painful recall (NRS 1-3) of the procedure (n/N) (\%) & $2(0.9)$ \\
\hline Reported painful recall (NRS $\geq 4)$ of the procedure (n/N) (\%) & $1(0.4)$ \\
\hline Reported pain at the time of propofol injection & $13(5.8)$ \\
\hline Reported pain at the site of the defipads after the procedure (n/N) (\%) & $15(6.4)$ \\
\hline Reported muscle pain after the procedure (n/N) (\%) & $6(0.9)$ \\
\hline
\end{tabular}


[2-15]. These studies have severe limitations. For example, Swann et al., [4] described $7.4 \%$ recall in 41 cardioversions and 84 orthopaedic reductions with a wide range of drug combinations used. Kaye et al. [17] and Valtonen et al. [10] described complete amnesia of ECV in all patients after sedation with propofol. However, in none of these studies recall was the primary outcome. The presence of recall was not routinely noted, which can result in an underreporting of the incidence of recall. Furthermore, Valtonen et al., [10] sedated 15 patients undergoing ECV with a mean dose of $2.5 \mathrm{mg} / \mathrm{kg}$ propofol. This dosage does not reflect current clinical practice. Miner et al. [8] described $16.5 \%$ recall of painful procedures after procedural sedation with propofol or propofol and alfentanil. However in this RCT only two patients underwent cardioversion, out of the 150 included patients. Furthermore, in most studies addressing recall supplemental opioid were added to propofol sedation $[5,6,8,10,12-15]$.

The evidence for the use of opioids in conjunction with propofol to prevent pain is limited and controversial. The studies that added opioids to propofol in short painful procedures showed similar incidences of recall compared to studies in which procedures were perfomed with propofol as a sole agent [2-15]. Propofol and remifentanil have shown to have a synergistic effect in terms of controlling a response to noxious stimuli during the procedure [18]. The clinical significance of procedural pain that a patient experiences, but cannot recall later, remains unclear [19]. Miner et al. [19] compared stress markers in short painful procedures in the emergency department in sedation with propofol with and without alfentanil in a small pilot study with 20 patients. Supplemental alfentanil did not appear to influence markers of physiologic stress compared to propofol as a sole agent. Furthermore, studies have demonstrated that administering opioids in addition to propofol may even contribute to increased rates of respiratory depression and clinical interventions [8,13,14,20-23].

In our study complete amnesia of the ECV was observed in $98.7 \%$ of the patients sedated with propofol, $1.9 \%$ did recall a feeling of slight discomfort (NRS 1-3) during the procedure. Based on the literature and our findings we do not recommend additional opioids to propofol sedation in ECV. Propofol provides good sedation and retrograde amnesia for the procedure. In previous literature opioids have not proven to be beneficial in ECV. On the contrary, opioid combined with propofol can increase the likelihood of adverse events $[9,10]$.

\section{Pain at the site of injection, defipads and muscle pain}

The secondary outcomes of this study were pain at the time of propofol injection and pain at the site of the defipads. In our study we $5.8 \%$ of the patients experienced injection pain. Literature describes pain of propofol injection in $2-33 \%$ of the patients $[7,12,22]$ The administration of intravenous lidocaine is found to prevent discomfort with $60 \%$ of the time with a rubber tourniquet in place 30 to 120 seconds before propofol administration [17]. Musclepain, burning sensations or pain at the side of the defipads was reported by $7.3 \%$ of the patients.

\section{Study design and limitations}

The variance to the dosage of propofol can be seen as a limitation of this study. However, our aim was to determine the incidence of painful recall in propofol sedation in current clinical practice. In current clinical practice careful titration of propofol until adequate sedation is achieved is preferred above the large boluses. Titration of propofol is known to prevent profound hypotension and oxygen desaturation $[1,5,11,12]$. Although the doses of propofol may appear relative 'light' for the patient's weight $(1.1 \mathrm{mg} / \mathrm{kg})$ compared to previous studies, adequate sedation with retrograde amnesia was achieved in $97.8 \%$ of the patients.

\section{Conclusion}

In this prospective multicenter study, painful recall of the ECV with NRS $\geq 4$ was found in $0.4 \%$ of the patients. Propofol as a sole agent provided effective sedation and retrograde amnesia in $99.6 \%$ of the patients. Our findings support that additional opioids to propofol sedation in ECV are not indicated to prevent painful recall.

\section{References}

1. Lewis SR, Nicholson A, Reed SS, Kenth JJ, Alderson P, et al. (2015) Anaesthetic and sedative agents used for electrical cardioversion. Cochrane Database of Syst Rev 3: CD01082. [Crossref]

2. Burton JH, Miner JR, Shipley ER, Strout TD, Becker C, et al. (2006) Propofol for emergency department procedural sedation and analgesia: a tale of three centers. Acad Emerg Med 13: 24-30. [Crossref]

3. Gottschalk A, Smith DS (2001) New concepts in acute pain therapy: preemptive analgesia. Am Fam Physician 63: 1979-1984. [Crossref]

4. Swann A, Williams J, Fatovich DM (2007) Recall after procedural sedation in the emergency department. Emerg Med J 24: 322-324. [Crossref]

5. Kalogridaki M, Souvatzis X, Mavrakis HE, Kanoupakis EM, Panteli A, et al. (2011) Anaesthesia for cardioversion: a prospective randomised comparison of propofol and etomidate combined with fentanyl. Hellenic J Cardiol 52: 483-488. [Crossref]

6. Zed PJ, Abu-Laban RB, Chan WW, Harrison DW (2007) Efficacy, safety and patient satisfaction of propofol for procedural sedation and analgesia in the emergency department: a prospective study. Can J Emerg Med 9: 421-427. [Crossref]

7. Akcaboy ZN, Akcaboy EY, Altinoren B, Karabulut E, Gogus N (2007) Adding remifentanil to propofol and etomidate in cardioversion anesthesia. Saudi Med J 28 1550-1554. [Crossref]

8. Parlak M, Parlak I, Erdur b, Ergin A, Sagiroglu A (2006) Age effect on efficacy and side effects of two sedation and analgesia protocols on patients going through cardioversion: a randomized clinical trial. Acad Emerg Med 13: 493-499.

9. Miner JR, Gray RO, Stephens D, Biros MH (2009) Randomized clinical trial of propofol with and without alfentanil for deep procedural sedation in the emergency department. Acad Emerg Med 16: 825-834. [Crossref]

10. Valtonen M, Kanto J, Klossner J (1988) Anaesthesia for cardioversion: a comparison of propofol and thiopentone. Can J Anaesth 35: 479-483. [Crossref]

11. Dworkin RH, Turk DC, Farrar JT, Haythornthwaite JA (2005) Core outcome measures for chronic pain clinical trials: IMMPACT recommendations. Pain 1: 9-19.

12. Gottschalk A, Smith DS (2001) New concepts in acute pain therapy: preemptive analgesia. Am Fam Physician 63: 1979-1984. [Crossref]

13. American Society of Anesthesiologists (ASA). Statement on the Safe Use of Propofol (amended October 15, 2014). www.asahq.org/For-Healthcare-Professionals/

14. Canessa R, Lema G, Urzúa J, Dagnino J, Concha M (1991) Anesthesia for elective cardioversion: a comparison of four anesthetic agents. J Cardiothorac Vasc Anesth 5: 566-568. [Crossref]

15. Miner JR, Bachman A, Kosman L, Teng B, Heegaard W, et al. (2005) Assessment of the onset and persistence of amnesia during procedural sedation with propofol. Acad Emerg Med 12: 491-496. [Crossref]

16. Miner JR, Burton JH (2007) Clinical practice advisory: emergency department procedural sedation with propofol. Ann Emerg Med 50: 182-218. [Crossref]

17. Jan KT, Wang KY, Lo Y, Lu BK, Liu K (1995) Anesthesia for elective cardioversion a comparison of thiopentone and propofol. Acta Anaesthesiol Sin 33: 35-39. [Crossref]

18. Kehlet H (1989) Surgical stress: the role of pain and analgesia. Br J Anaesth 63: 189195. [Crossref]

19. Miner JR, Moore JC, Plummer D, Gray RO, Patel S, et al. (2013) Randomized clinical trial of the effect of supplemental opioids in procedural sedation with propofol on serum catecholamines. Acad Emerg Med 20: 330-337. [Crossref] 
20. Messenger DW, Murray HE, Dungey PE, van Vlymen J, Sivilotti ML (2008) Subdissociative-dose ketamine versus fentanyl for analgesia during propofol procedural sedation: a randomized clinical trial. Acad Emerg Med 15: 877-886. [Crossref]

21. Moerman AT, Struys MM, Vereecke HE, Herregods LL, De Vos MM, et al. (2004) Remifentanil used to supplement propofol does not improve quality of sedation during spontaneous respiration. J Clin Anesth 16: 237-243. [Crossref]
22. Black E, Campbell SG, Magee K, Zed PJ (2013) Propofol for procedural sedation in the emergency department: a qualitative systematic review. Ann Pharmacother 47: 856868. [Crossref]

23. Picard P, Tramèr MR (2000) Prevention of pain on injection with propofol: a quantitative systematic review. Anesth Analg 90: 963-969. [Crossref]

Copyright: (C2018 Van Winden DFM. This is an open-access article distributed under the terms of the Creative Commons Attribution License, which permits unrestricted use, distribution, and reproduction in any medium, provided the original author and source are credited. 